\title{
Recombinant ACE2- opportunities and challenges in COVID 19 treatment.
}

\author{
Rahul Sandhu ${ }^{1}$ and Mandeep Kaur ${ }^{2}$ \\ ${ }^{1}$ Armed Forces Medical College \\ ${ }^{2}$ National Institute of Virology
}

May 6, 2020

\begin{abstract}
Severe acute respiratory syndrome coronavirus 2 (SARS-COV2) emerged from Wuhan, China at the end of December and spread rapidly around the globe with a higher degree of lethality reported than the endemic coronaviruses. Angiotensin convertase enzyme 2 (ACE2) host cell receptors mediate viral entry by binding to spike S protein of SARS-CoV2. ACE2 binding in viral pathogenesis has opened newer avenues for COVID-19 treatment with ACE2 at the center stage. Recombinant human ACE2 (rhACE2) protein has already shown therapeutic potential for vast array of therapeutic indications. In SARS-CoV2, the competitive binding of viral S protein with circulating ACE2 causing virus neutralization and sparing host ACE2 receptors is being proposed as the potential therapeutic mechanism in COVID-19. Though rhACE2 has moved into clinical trials in SARS-CoV2 patients, there are still some research gaps including lack of sufficient data about the proven efficacy of rhACE2 from in vitro and in vivo studies.
\end{abstract}

\section{Introduction}

The emergence of a novel strain of coronavirus severe acute respiratory syndrome coronavirus 2 (SARS COV2) from Wuhan, China has brought the whole world down to their knees. Belonging to the $\beta$ lineage of coronaviruses, family Coronaviridae, order Nidovirales and genus sarbecovirus, this RNA virus with the largest genome $(26-32 \mathrm{~kb})$ has led to a global outbreak of respiratory disease, with over $3,356,634$ human cases and 7\% mortality. The worst affected region in terms of morbidity is United States of America with 1,104,324 cases and as far as mortality is concerned, the European region is badly affected with reported mortality of more than $10 \%$ ([?]13\% reported from Italy, France and United Kingdom). [1] [https://www.worldometers.info/coronavirus on 01 May 2020].

In current scenario when specific therapeutics are not available, and applying travel restrictions, patient isolation, and providing supportive medical care remain the mainstay for patient management, angiotensin convertase enzyme 2 (ACE2), a host cell surface receptor has garnered much attention as it acts as a key receptor for attachment to the spike glycoprotein of SARS-CoV2 to initiate viral infection. [2] In this article, we will discuss the potential application of recombinant human soluble ACE2 (rhACE 2) as therapy in COVID-19 patients and the challenges that can hinder its clinical use.

ACE2, a type 1 transmembrane glycoprotein was first discovered in the year 2000. It is present widely in human tissues but relatively high level of expression has been observed in respiratory epithelial cells, alveolar cells type I and II, oral cavity, kidney, testis, small intestines, arterial and venous endothelial cells and arterial smooth muscle cells. Glomerular tubules show low ACE2 expression, while glomerular mesangial and glomerular endothelial cells including Kupffer cells and hepatocytes, spleen, thymus, lymph nodes, bone marrow, and B and T lymphocytes and macrophages don't possess ACE2 activity. Tissue ACE2 activity has 
been observed to be higher than its plasma activity and traditional angiotensin-converting enzyme inhibitors (ACEIs) don't inhibit its function. [3]

ACE2 is an essential part of counter regulatory axis of renin angiotensin system (RAS), and has extensive vascular and organ protection functions in hypertension, diabetes, cardiovascular disease, and ARDS. ACE2 has catalytic activity against both Angiotensin II (Ang II) and Angiotensin I (Ang I) but its affinity for Angiotensin II is 400 times higher than that for Ang I. [3] ACE2 hydrolyzes Ang II and Ang I generating angiotensin 1-7 (Ang 1-7) and angiotensin 1-9, respectively. Ang 1-7 binds to the Mas receptor to exert antiinflammatory and anti-remodeling effects antagonizing the pro-inflammatory, pro-proliferative, and fibrotic effects caused by Angiotensin II. [3]

In SARS-COV2, ACE2 acts as co-receptors facilitating viral entry by interaction between receptor-binding domain (RBD) of the S1 subunit on viral spike glycoproteins with the ectodomain of ACE2. The viral endocytosis leads to the downregulation of ACE2 receptors either through direct effect (rapid apoptosis) or indirectly by increasing the ADAM metallopeptidase domain 17 (ADAM 17) induced catalytic cleavage of ectodomain of ACE2 releasing it into the circulation. [4]

The increased Ang II acts on AT1 receptor and further upregulate ADAM17 in a well characterized positive feedback loop that lead to shedding of its regulator ACE2 and downstream extracellular signal regulated kinase $($ ERK)/p38 mitogen activated protein (MAP) kinase signaling pathways. ADAM17 also mediates the liberation of membrane bound precursors of $\mathrm{TNF} \alpha, \mathrm{IFN}-\gamma$, and IL-4 pro-inflammatory cytokines into the circulation, justifying its alternative name i.e. tumor necrosis factor converting enzyme (TACE) (figure 1). This cytokine storm and the reduced ACE2 activity may be associated with the disease severity in SARS-CoV2 infection. [4]

ACE2 expressed in type II alveolar epithelial cells primarily function to prevent alveolar collapse which are critical to the gas exchange function of the lung by producing surfactant that reduces surface tension. Injury to these cells in SARS-COV2 infection could explain the severe lung injury and ARDS observed in COVID19 patients. [3] Despite the predominance of respiratory symptoms in SARS-COV2, the acute cardiac and kidney injuries, myocarditis, arrhythmias, and gut and liver abnormalities occurring in COVID-19 patients are consistent with the widespread expression of ACE2. [2]

The enzymatic conversion of the proinflammatory, profibrotic vasoconstrictor Ang II into Ang 1-7 that exerts anti-inflammatory, antifibrotic and cardioprotective vasodilator makes the soluble ACE2 receptor a reasonable therapeutic approach in settings where Ang II is involved in the pathologic mechanism (like in SARS-CoV2). [1] Recombinant soluble ACE2 has already shown potential for a vast array of therapeutic indications. It has shown good safety and tolerability profile in phase 1 and 2 trials done in healthy volunteers and in patients suffering from ARDS and single doses (100 and $1200 \mathrm{mg} / \mathrm{kg}$ rsACE2) administered intravenously in healthy volunteers had revealed a plasma half-life of 10 hours and peak plasma concentration to be up to $20 \mathrm{mg} / \mathrm{ml}(223 \mathrm{nM})$ in the highest-dose cohort. [5]

In SARS-CoV2, the proposed use of soluble ACE2 (sACE2) receptor is that it would bind to the viral S protein thereby neutralize the virus and prevent virus mediated host cell receptor downregulation (figure 1). SARS-COV2 and SARS CoV spike proteins share $76.5 \%$ identity in amino acid sequences and, importantly, there is a high degree of homology between spike proteins of SARS-COV2 and SARS-CoV. [6] Soluble ACE2 receptor was demonstrated to inhibit SARS CoV binding to cells in culture suggesting this strategy to be very promising against SARS-CoV2 too. [7] Apart from S protein neutralizing agent, an additional advantage of using recombinant ACE2 in SARS-CoV2 would be its role in treatment of pneumonia in COVID-19 patients. $[6]$

ACE2-Fc, an immunoadhesin format of sACE2 where sACE2 is fused to immunoglobulin Fc domain appear more convincing and effective option by extending the lifespan of the circulating molecule while retaining the effector functions of the Fc domain, thereby allowing recruitment of dendritic cells, macrophages, and natural killer cells through the CD16 receptor against viral particles or infected cells. Moreover, anin vitro study using sACE2 fused to the Fc portion of immunoglobulin has reported successful neutralization of 


\section{SARS-CoV2. [7]}

The availability of safe rhACE2 formulation gave researchers the encouragement and motivation to rapidly start a pilot trial of rhACE2 in patients suffering from severe COVID-19 (Clinicaltrials.gov \#NCT04287686). [6] Nevertheless, as far as rhACE2 therapy is concerned, the results of the ongoing trials and upcoming clinical trials conducted on large number of patients would provide the definitive and convincing evidence for its use in COVID-19 patients.

Despite good safety profile of recombinant human ACE2, there are research gaps that need to be addressed before it is approved for clinical use in SARS-CoV2 infection. The challenges are that the understanding about SARS-CoV2 pathogenesis is still in evolving and in its naïve stage. Thereby, the possibility of virus making use of other co-receptors/auxiliary proteins or even other mechanisms for entry into the cells cannot be ruled out. [1] For pathophysiology of COVID 19, till date the biochemical evidence about the RAS dysregulation in COVID-19 is lacking, only a single study from China had reported raised levels of Angiotensin II in all 12 COVID-19 patients as compared to healthy controls indicating that RAS overactivation could be responsible for severity of infection. Clinical data about the RAS metabolite levels (i.e. Aldosterone, Angiotensin II) and sACE2 in serial patient blood samples of COVID-19 can provide crucial information to answer questions on disease predisposition, its progression and on the mechanisms affected by the viral infection. [8]

In a recent study conducted by Montiel et al, the use of the clinical-grade human ACE2 molecule - but not mouse sACE2 was found to significantly inhibit SARS-CoV2 infections and reduction of viral load by a factor of 1,000-5,000 was observed when virus was grown in Vero E6 cells. They further found that engineered human blood vessel organoids and human kidney organoids can be readily infected, which can significantly be inhibited by rhACE2 at an early stage of infection. But they failed to perform similar studies on human lung tissue which is the main organ affected in SARS-CoV2 infection. Hence in vitrostudies testing sACE2 efficacy against SARS-CoV2 in different human cell lines followed by in vivo experiments should be carried out to have better understanding of the multi-organ involvement in SARS-CoV2 infection. It would be also be interesting to note the viral pathogenesis in ACE2 deficient individuals or similar in vitroexperiments in cell lines with reduced ACE2 expression can provide more understanding. [9]

The cross transmission of virus between bats and/ or other intermediate hosts and humans is the result of structural variations of the viral spike protein and the sequence variation in animal host ACE2 receptors may also contribute towards viral susceptibility and/or resistance. Although many ACE2 variants have shown a similar binding affinity for SARS-CoV2 spike protein, ACE2 alleles, rs73635825 (S19P) and rs143936283 (E329G) have showed noticeable variations in their intermolecular interactions with the viral spike protein. Hence, it is conceivable that under new selection pressure as offered by SARS-CoV2, these alleles may undergo positive selection and can have an impact on the progression of clinical trials testing the utility of recombinant ACE2 as COVID-19 therapy. [10] Thus, largescale and multiple tissue-level analysis of singlecell RNA sequence would provide precise information about the expression analysis of ACE2 in different populations and in turn help in selecting the candidates for rhACE2 therapy.

\section{Conclusion}

For this rapidly evolving pandemic, the development of a specific antiviral agent against the virus is of course what is needed, but series of preclinical trials before any novel agent becomes available for clinical use makes it an improbable option. Thus, the need of the hour would be to assess the available armoury of drugs and use them in defence against the current enemy.

\section{Acknowledgements}

Conflict of interest- Nothing is declared

Source of funding -None

\section{References}


1. Michael Letko M, Marzi A, Munster V. Functional assessment of cell entry and receptor usage for SARS-CoV-2 and other lineage B Betacoronaviruses. Nat Microbiol 2020;5:562-9.

2. Wang K, Gheblawi M, Oudit GY. Angiotensin Converting Enzyme 2: a double-edged sword. Circulation. 2020. doi: 10.1161/CIRCULATIONAHA.120.047049

3. Cheng H, Wang Y, Wang GQ. Organ-protective effect of angiotensin-converting enzyme and its effect on the prognosis of COVID-19. J Med Virol 2020;1-5. https://doi.org/10.1002/jmv.25785.

4. Fu Y, Cheng Y, Wu Y. Understanding SARS-CoV-2 mediated inflammatory responses: from mechanisms to potential therapeutic tools. Virol Sin Matrch 2020. doi.org/10.1007/s12250-020-00207-4.

5. Guignabert C, de Man F, Lombes M. ACE2 as therapy for pulmonary arterial hypertension: the good outweighs the bad. Eur Respir J 2018;51:1800848.

6. Zhang H, Penninger JM, LiY, Zhong N, Slutsky AS. Angiotensin-converting enzyme 2(ACE2) as a SARS-CoV-2 receptor: molecular mechanisms and potential therapeutic target. Intensive Care Med 2020;46:586-90.

7. Kruse RL. Therapeutic strategies in an outbreak scenario to treat the novel coronavirus originating in Wuhan, China [version 2; peer review: 2 approved] F1000 Research 2020; 9:72.

8. Liu Y, Yang Y, Zhang C, Huang F, Wang F, Yuan J, et al. Clinical and biochemical indexes from 2019-nCoV infected patients linked to viral loads and lung injury. Sci China Life Sci 2020;63:364-74. doi.org/10.1007/s11427-020-1643-8.

9. Monteil V, Kwon H, Prado P, Hagelkruys A, Wimmer RA, Stahl M, et al. Inhibition of SARS-CoV2 infections in engineered human tissues using clinical-grade soluble human ACE2. Cell 2020. pii: S0092-8674(20)30399-8. doi: 10.1016/j.cell.2020.04.004.

10. Hussain M, Jabeen N, Raza F, Shabbir S, Baig AA, Amanullah A, et al. Structural variations in human ACE2 may influence its binding with SARS-CoV-2 spike protein. J Med Virol 2020;1-7. doi.org/10.1002/jmv.25832.

\section{Figure Legend}

Figure 1: SARSCoV2 and ACE2 interaction and effect of recombinant soluble ACE2 on viral binding.

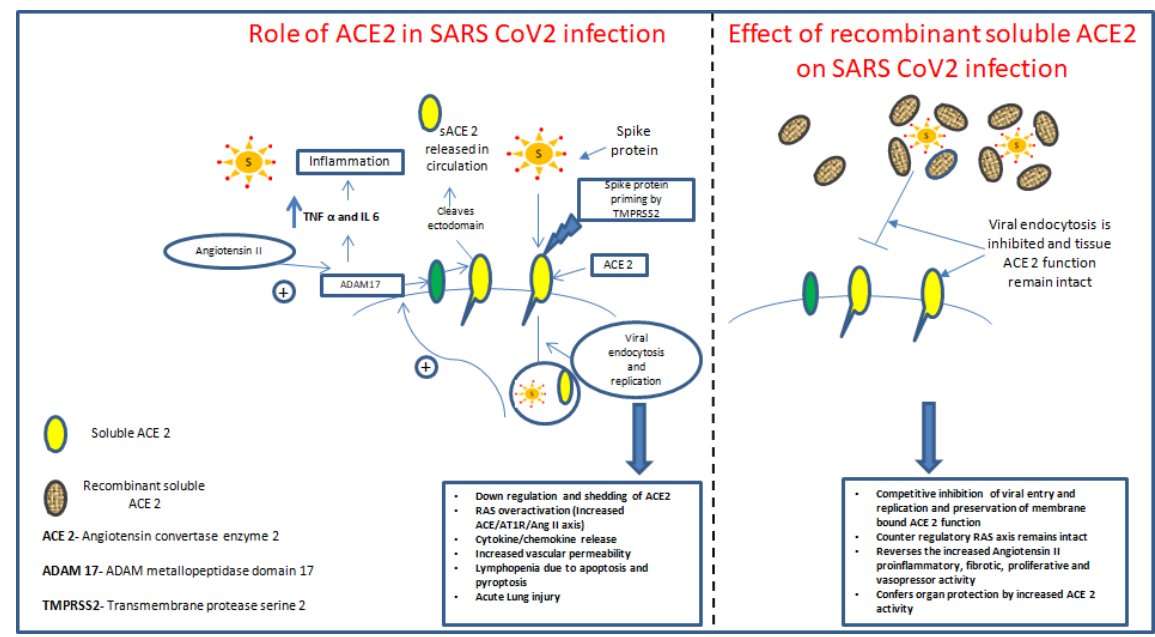

